\title{
Temperature Dependence of Electron Tunneling between Two Dimensional Electron Gas and Si Quantum Dots
}

Yoko Sakurai $^{1}$, Jun-ichi Iwata ${ }^{2,7}$, Masakazu Muraguchi ${ }^{3,7}$, Yasuteru Shigeta ${ }^{4,7}$, Yukihiro

Takada $^{1}$, Shintaro Nomura ${ }^{1}$, Tetsuo Endoh ${ }^{3,7}$, Shin-ichi Saito ${ }^{5}$, Kenji Shiraishi ${ }^{1,2,7}$,

Mitsuhisa Ikeda ${ }^{6}$, Katsunori Makihara ${ }^{6}$, and Seiichi Miyazaki ${ }^{6}$

${ }^{1}$ Graduate School of Pure and Applied Sciences, University of Tsukuba, Tsukuba, Ibaraki 305-8571, Japan

${ }^{2}$ Center for Computational Sciences, University of Tsukuba, Tsukuba, Ibaraki 305-8577, Japan

${ }^{3}$ Center for Interdisciplinary Research, Tohoku University, Sendai, Miyagi 980-8578, Japan

${ }^{4}$ Institute of Picobiology, Graduate School of Life Science, University of Hyogo

${ }^{5}$ Central Research Laboratory, Hitachi Ltd., Kokubunji, Tokyo 185-8601, Japan

${ }^{6}$ Graduate School of Advanced Science and Matter, Hiroshima University, Higashihiroshima, Hiroshima 739-8530, Japan

${ }^{7}$ CREST, Japan Science and Technology Agency, Kawaguchi, Saitama 332-0012, Japan

Quantum mechanical electron tunneling has potential applications in both science and technology, such as flash memories in modern LSI technologies and electron transport chains in biosystems. Although it is known that one-dimensional quantum electron 
tunneling lacks temperature dependence, the behavior of electron tunneling between different dimensional systems is still an open question. Here, we investigated the electron tunneling between a two-dimensional electron gas (2DEG) and zero-dimensional $\mathrm{Si}$ quantum dots and discovered an unexpected temperature dependence: At high temperature, the gate voltage necessary for electron injection from 2DEG to Si quantum dots becomes markedly small. This unusual tunneling behavior was phenomenologically explained by considering the geometrical matching of wave functions between different dimensional systems. We assumed that electron tunneling would occur within a finite experimental measurement time. Then, the observed electron tunneling is explained only by the contributions of wave packets below the quantum dot with a finite lifetime rather than the ordinary thermal excited states of 2DEG. 


\section{Introduction}

Quantum mechanical electron tunneling has crucial applications in science and technology [1]. Electron wave functions can tunnel through a potential barrier by overlap between initial and final state wave functions, and quantum mechanical electron tunneling has made possible modern semiconductor technologies such as flash memories in very large scale integration (VLSI) devices [2,3]. The basic tunneling treatment has apparently been established, and tunneling modes can typically be classified as resonant, Fowler-Nordheim, or direct tunneling [4]. However, conventional treatments of electron tunneling imply that the simple one-dimensional overlap integral between the initial and final state wave functions determines the tunneling probabilities, assuming that both wave functions and the potential barrier infinitely extend in the direction perpendicular to the propagation direction. Furthermore, quantum electron tunneling has been known to lack temperature dependence, as long as the tunneling mode is direct. In fact, Wakabayashi et al. reported no temperature dependence of subthreshold characteristics in transistors with very small gate lengths [5]. Here, we investigated the electron tunneling from a two-dimensional electron gas (2DEG) to zero-dimensional Si quantum dots (QDs) and discovered an unexpected temperature dependence. The gate voltage necessary for electron injection from 2DEG to Si QDs becomes markedly small, at high temperature. This was phenomenologically explained by the temporal and spatial fluctuations in a 2DEG, originating from the geometrical matching of wave functions between different dimensional initial and final states, assuming that the electron tunneling occurred within a finite experimental measurement time. 
Electron tunneling between different dimensional systems commonly occurs in real situations. Tunneling from two-dimensional to zero-dimensional systems is a well-known phenomenon. For example, tunneling currents through a dielectric barrier via zerodimensional quantum dots or defects have been studied extensively from both scientific and technological view points [6-10]. Moreover, even in biosystems, electron transfer between an extended $\mathrm{p}$ orbital in a porphyrin ring and a localized atomic orbital of a metallic ion is expected to be related to the physiological functions of proteins [11]. The deep understanding of electron tunneling between different dimensional systems should lead to significant progress in a wide range of scientific and technological areas.

For tunneling phenomena between different dimensional systems, the geometrical matching of wave functions, which is neglected in conventional one-dimensional tunneling treatments, is important [12], in addition to the electron-energy matching between initial and final states (the resonant condition), assuming that tunneling events occur within a finite experimental measurement time (Fig. 1). Electron tunneling occurs only when the electron wave functions are sufficiently wave-packet-like just below the zero-dimensional sites caused by the thermal fluctuation. However, if the dimensions of the initial and final states are the same, no modification of the initial state electron wave functions is necessary to achieve geometrical matching, so only the resonant condition governs the electron tunneling. To achieve the above-mentioned geometrical matching, the wave-packet-like modification of wave functions by the thermal fluctuation should play crucial roles in electron tunneling. This implies that the electron tunneling between different dimensional systems generally shows temperature dependence, unlike that between the same 
dimensional systems. However, tunneling phenomena have not yet been considered in light of the differences in the initial and final state dimensions.

The organization of this paper is as follows. In $\S 2$, we describe our Si quantum dot samples. We show the main results and discussion in $\S 3$, and the conclusions are given in $\S 4$.

\section{Samples}

In this study, we considered a sample in which Si QDs were weakly coupled to a 2DEG through a 3.5-nm-thick $\mathrm{SiO}_{2}$ barrier layer [13]. Figures 2(a) and 2(b) show schematics of the Si QD-2DEG coupled sample and transmission electron microscopy (TEM) images of the Si QD, respectively. The Si QD was hemispherical in shape, and the estimated average height, diameter, and density were $5.1 \mathrm{~nm}$, approximately $10 \mathrm{~nm}$, and $1.7 \times 10^{11} \mathrm{~cm}^{-2}$, respectively. To investigate the characteristics of the Si QD, we examined the electronic structures of a spherical Si QD model by first-principles calculations within the local density approximation (LDA) and norm-conserving pseudo potential [14-18]. In the calculation, the real space formalism was used for large-scale calculations [19-21]. The calculated density of states is shown in Fig. 2(c). As shown, the spherical Si QD of $5 \mathrm{~nm}$ diameter has almost continuous energy levels except in the band-edge regions, although it was sufficiently small to reveal a zero-dimensional characteristic from a geometrical viewpoint. In particular, the average energy level splitting of the spherical Si cluster model was $0.5-1.0 \mathrm{meV}$ in the energy region of approximately $1.4 \mathrm{eV}$ above the conduction band bottom, which corresponded to the typical energy range of electron injection from the 2DEG to Si QDs in our experiments. Accordingly, the resonant condition was almost 
always satisfied regardless of the applied gate voltage in our experiments with the typical temperature range between 100 and $240 \mathrm{~K}$. The resonant condition, however, was commonly the most important for electron transfer from the 2DEG to QDs in previous experiments, which generally used smaller QDs with large energy spacing between quantized levels [7-10]. Our current sample was designed such that the wave-packet-like modification of the 2DEG wave function induced by the thermal fluctuation played the most important role in electron injection from the 2DEG to Si QDs, instead of the resonant condition. The electron tunneling from two-dimensional to one-dimensional systems could also be examined in our $\mathrm{Si}$ QD samples, as a high-quality $2 \mathrm{DEG}$ formed at $\mathrm{Si} / \mathrm{SiO}_{2}$ interfaces $[22,23]$.

\section{Results and Discussion}

The electron injection currents from the 2DEG to Si QDs were measured as functions of the gate voltage $\left(V_{\mathrm{G}}\right)$ between an $\mathrm{n}^{+}$-poly-Si gate electrode and a p-Si substrate. Figure 3 shows the observed displacement electron currents as functions of $V_{\mathrm{G}}$ and temperature (T). Clear peaks corresponding to electron injections from the 2DEG to Si QDs appeared at +2 to $+4 \mathrm{~V}$ to gate. The appearance of multicurrent peaks was explained on the basis of the Coulomb blockade effect. Surprisingly, the gate voltages necessary for the electron injection from the 2DEG to Si QDs had a clear temperature dependence, although the

electron injection currents through a sufficiently thin $3.5 \mathrm{~nm} \mathrm{SiO}_{2}$ barrier layer have conventionally been described using a temperature-independent direct tunneling scheme. The observed gate voltages necessary for the electron injection markedly changed from 3.7 to $2.2 \mathrm{~V}$ as the temperature increased from 120 to $240 \mathrm{~K}$. 
The direct tunneling current through a simple energy barrier has been suggested to have no temperature dependence on the basis of the quantum mechanical consideration [1]. This indicated that a novel mechanism originating from the difference in dimensions between the 2DEG and Si QD governed the temperature dependence. To explain this unexpected finding, we have made a basic phenomenological assumption that the tunneling event should occur within a finite experimental time. On the basis of the above assumption, only the highly localized 2DEG wave functions can contribute to tunneling events from the 2DEG to the QDs. The wave functions correspond to the "sufficiently localized" wavepacket-like ones, whose energies are larger than a critical value and play crucial roles in the elessctron tunneling between different dimensional systems.

We investigated the energy of a Gaussian-type wave packet as a function of a halfwidth of a Gaussian-type wave packet to qualitatively explain the above unusual temperature dependence. As shown in Fig. 4, Gaussian-type wave packets with a narrow half-width have high energies. Accordingly, "sufficiently localized" wave-packet-like wave functions in a 2DEG can be generated easily at high temperatures owing to thermal fluctuation. Accordingly, the gate voltages necessary for electron injection $\left(V_{\mathrm{G}}\right)$ decrease with the increase in temperature, qualitatively reproducing the present unexpected temperature dependence. Although the physical origin of the present phenomenological assumption is unclear, there is a possibility that a dynamical electron correlation may cause a critical energy value of wave-packet-like 2DEG wave functions, which can contribute to electron tunneling between 2DEG and Si QDs [24]. However, further careful and detailed discussions are necessary to clarify the present unexpected phenomena. 
Next, we discuss the temperature dependence of the multicurrent peaks in I-V characteristics which we attributed to Coulomb blockade. The differences between multicurrent peaks are about 100-200 meV; the difference between peak 2 and peak 1 and that between peak 3 and peak 2 are about 200 and $100 \mathrm{meV}$, respectively. Moreover, these differences have a small temperature dependence, although the peak width is broadened at high temperature.

These values of 100-200 meV are much larger than the difference between $5 \mathrm{~nm}$ diameter dot levels of approximately $0.5 \mathrm{meV}$, as shown in Fig. 2(c). Discrete levels of this dot $(\sim 0.5 \mathrm{meV})$ can only be detected in very low temperature experiments. Our firstprinciples calculations also showed that the charging energy of the $5 \mathrm{~nm}$-diameter dot is about $100 \mathrm{meV}$ if the dot is embedded in $\mathrm{SiO}_{2}$ with a dielectric constant of 3.9. Thus, we conclude that the observed multicurrent peaks essentially originate from the Coulomb blockade effect. However, this Coulomb blockade effect is a slightly different from that observed in a single electron transistor (SET). In our sample, electrons are only injected from 2DEG to Si QDs. On the other hand, electrons tunnel through QDs to drain electrodes in SET samples. Thus, one peak essentially corresponds to one electron injection into Si QDs in our sample. Thus, even in the situation in which tunneling events are likely to occur at high temperature, a marked peak height increase does not occur.

However, many experiments have not shown a clear temperature dependence. For example, the reported electron current characteristics through QDs in SET structures have little temperature dependence [7-10]. What was the origin of the discrepancy between our results, which showed a clear temperature dependence, and those of previous reports, which showed little temperature dependence? A notable difference was that previous studies used 
smaller QDs by targeting the systematic consideration of the coupling between discrete QD levels and the 2DEG. Accordingly, the resonant condition rather than the wave-packet-like modification of the 2DEG wave functions induced by the thermal fluctuation governed the tunneling currents, leading to little temperature dependence of $I-V$ characteristics. In our sample, however, the resonant condition was almost always satisfied regardless of the applied gate voltages, as shown by the first-principles calculations [Fig. 2(c)]. Accordingly, the thermal fluctuation of the 2DEG wave functions is crucial for the electron injection from the 2DEG to the zero-dimensional Si QDs. This implied that the difference in dimensions between the initial and final states was important for electron tunneling.

In short, the observed temperature dependence of tunneling currents originated from the non thermal-equilibrium nature of the 2DEG electrode, which has conventionally been treated as a reservoir in thermal equilibrium. The importance of the non thermalequilibrium nature of electrodes has been pointed out theoretically; the quantum reflection in a drain electrode plays a crucial role in one-dimensional ballistic transport $[25,26]$. Moreover, we expect that other types of thermal fluctuation effects in 2DEGs may be detected by further systematic investigations of electron tunneling between different dimensional systems even under resonant conditions.

\section{Conclusions}

We have found that electron tunneling between different dimensional systems showed an unexpected temperature dependence owing to the temporal and spatial fluctuations of electron wave functions in the initial states. This unexpected temperature dependence can be qualitatively reproduced on the basis of the phenomenological assumption that sufficiently wave-packet-like wave functions, whose energies are larger 
than the critical value, can contribute to the electron tunneling between different dimensional systems.

\section{Acknowledgements}

Computations were carried out using the PACS-CS supercomputer at the Center for Computational Sciences of the University of Tsukuba, Japan. This work was partially supported by a Grant-in-Aid for Scientific Research No. 18063003 from Ministry of Education, Culture, Sports, Science and Technology (MEXT).

[1] L. I. Schiff: Quantum Mechanics (McGraw-Hill, New York, 1949).

[2] G. E. Moore: Electron. Mag. 38 (1965) No. 8.

[3] T. Endoh R. Shirota S. Aritome, and F. Masuoka, IEICE Trans. Electron. E75-C (1992) 1351.

[4] E. H. Nicolian and J. R. Brews: MOS (Metal Oxide Semiconductor) Physics and Technology (Wiley, Hoboken, NJ, 2003), p. 232.

[5] H. Wakabayashi, T. Ezaki, M. Hane, S. Yamagami, N. Ikarashi, K. Takeuchi, T. Yamamoto, T. Mogami, T. Ikezawa, T. Sakamoto, and H. Kawaura: Tech. Dig. IEDM, 2004, p. 429.

[6] K. Torii, K. Shiraishi, S. Miyazaki, K. Yamabe, M. Boero, T. Chikyow, K. Yamada, H. Kitajima, and T. Arikado: Tech. Dig. IEDM, 2004, p. 129.

[7] U. Meirav, K. A. Kastner, and S. J. Wind: Phys. Rev. Lett. 65 (1990) 771. 
[8] Y. Takahashi, H. Namatus, K. Kurihara, K. Iwadate, M. Nagase, and K. Murase: IEEE Trans. Electron Devices 43, (1996) 1213.

[9] D. G. Austing, T. Honda, and S. Tarucha: Solid-State Electron. 40 (1996) 237.

[10] S. Sasaki, S. De Franceschi, J. M. Elzerman, W. G. van der Wiel, M. Eto, S. Tarucha, and L. P. Kouwenhoven: Nature 405 (2000) 764.

[11] S. F. Miller and G. T. Babcock: Chem. Rev. 96 (1996) 2889.

[12] M. Muraguchi, Y. Takada, S. Nomura, K. Shiraishi, and T. Endoh: Tech. Dig. Int. Meet. Future of Electron Devices, Kansai, 2009, p. 46.

[13] S. Miyazaki, Y. Hamamoto, E. Yoshida, M. Ikeda, and M. Hirose: Thin Solid Films 369 (2000) 55.

[14] P. Hohenberg and W. Kohn: Phys. Rev. 136 (1964) B864.

[15] W. Kohn and L. J. Sham: Phys. Rev. 140 (1965) A1133.

[16] N. Troullier and J. L. Martins: Phys. Rev. B 43 (1991) 1993.

[17] D. M. Ceperley and B. J. Alder: Phys. Rev. Lett. 45 (1980) 566.

[18] J. P. Perdew and A. Zunger: Phys. Rev. B 23 (1981) 5048.

[19] J. R. Chelikowsky, N. Troullier, and Y. Saad: Phys. Rev. Lett. 72 (1994) 1240.

[20] J. R. Chelikowski, N. Troullier, K. Wu, and Y. Saad: Phys. Rev. B 50 (1994) 11355.

[21] J.-I. Iwata, K. Shiraishi, and A. Oshiyama: Phys. Rev. B 77 (2008) 115208.

[22] T. Ando, A. B. Fowler, and F. Stern: Rev. Mod. Phys. 54 (1982) 437.

[23] S. V. Kravchenko and M. P. Sarachik: Rep. Prog. Phys. 67 (2004) 1.

[24] M. Muraguchi, Y. Takada, S. Nomura, and K. Shiraishi: Jpn. J. Appl. Phys. 47 (2008) 7807.

[25] K. Natori: J. Appl. Phys. 76 (1994) 4879. 
[26] S. Datta, F. Assad, and M. S. Lundstrom: Superlattices Microstruct. 23 (1998) 771. 
Figures

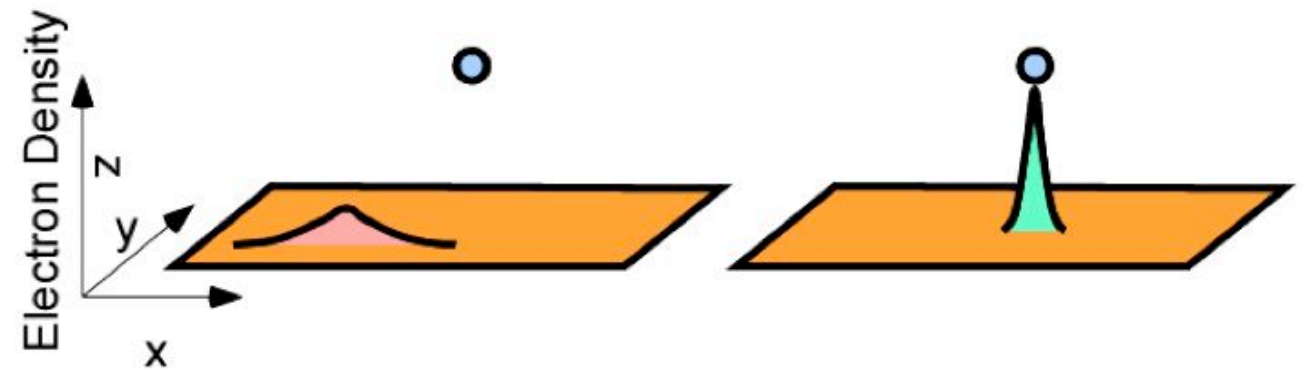

Fig. 1

Fig. 1. Schematic of electron tunneling from a two-dimensional system to a zerodimensional system. If the geometrical matching of the electron wave functions is not satisfied, electron tunneling from a two-dimensional to a zero-dimensional system does not occur (left). Electron tunneling can occur only when geometrical matching of the wave functions is achieved (right). 
(a)

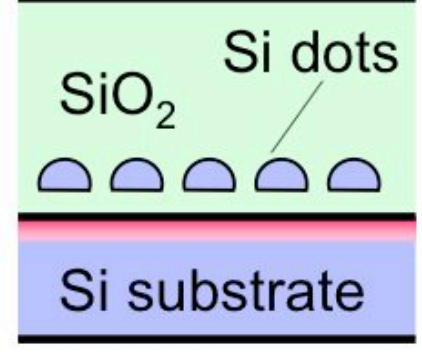

(b)

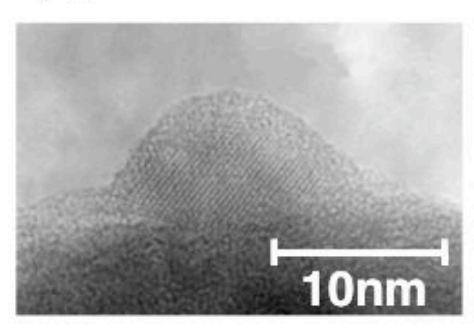

(c)

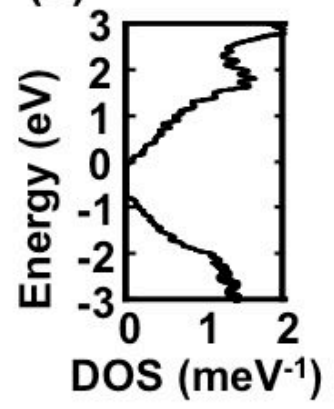

Fig. 2

Fig. 2. (a) Schematic of a sample of the Si quantum dot metal oxide semiconductor (MOS) capacitor. Si quantum dots were weakly coupled to a p-type Si substrate through a 3.5-nmthick $\mathrm{SiO}_{2}$ barrier layer. Si quantum dots were covered by a 7.5-nm-thick controlled $\mathrm{SiO}_{2}$ barrier layer and the electrode was a $300-n m-$ thick $\mathrm{n}^{+}$-poly-Si. The sample size was $0.5 \times$ $0.5 \mathrm{~mm}^{2}$. (b) Cross-sectional transmission electron microscopy (TEM) images of a $\mathrm{Si}$ quantum dot. Each Si dot had a hemisphere-like structure. (c) Calculated density of states (DOS) of a 5nm-diameter Si nanocluster. The energy of the conduction band bottom was set as zero. 
Fig. 3

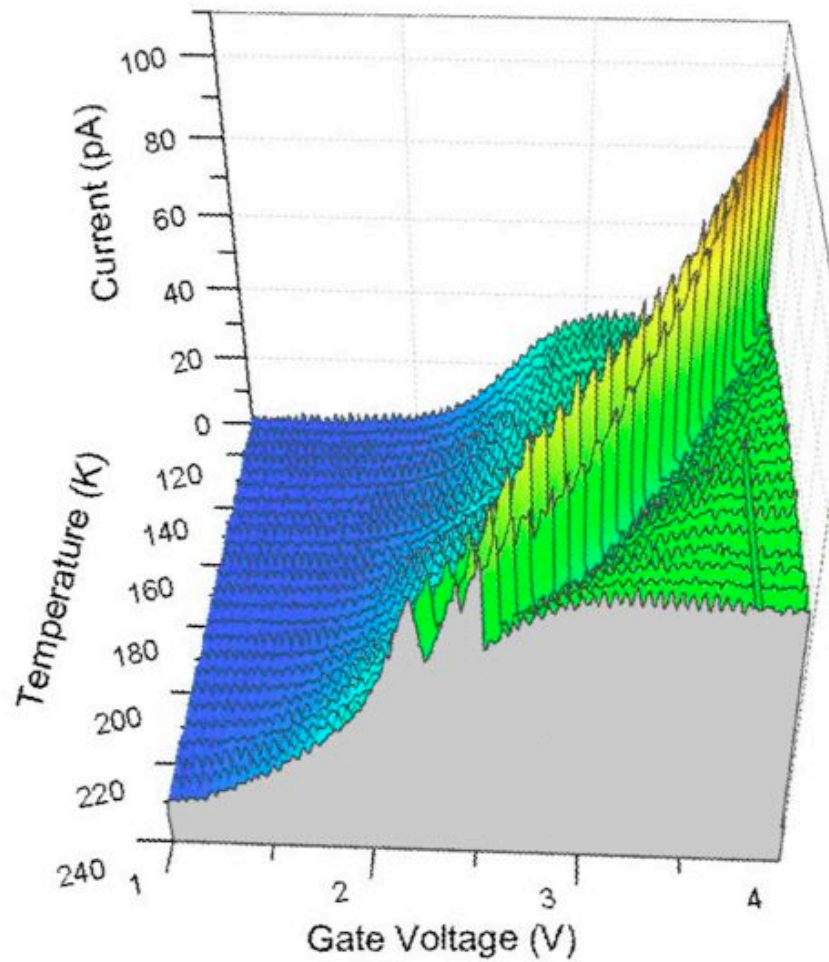

Fig. 3. Obtained displacement currents $(I)$ measured at different temperatures and the temperature dependence of the gate voltage necessary for electron injection obtained by theory and experiments. Displacement characteristics plotted as functions of the applied gate voltages between an $\mathrm{n}^{+}$-poly-Si electrode and a p-Si substrate $\left(V_{\mathrm{G}}\right)$ at temperatures $(\mathrm{T})$ between 120 and $240 \mathrm{~K}$. The $I-V_{\mathrm{G}}$ characteristics were measured using a two-terminal source meter (2635A, Keithley) with triax cables. The sample was located in a He-flow cryostat to stabilize the temperature. Each temperature gate voltage was swept with a 10 $\mathrm{mV}$ step between -4 and $4 \mathrm{~V}$, corresponding to the effective gate voltage between -2 and 6 $\mathrm{V}$, at a sweep rate of $63 \mathrm{mV} / \mathrm{s}$. 


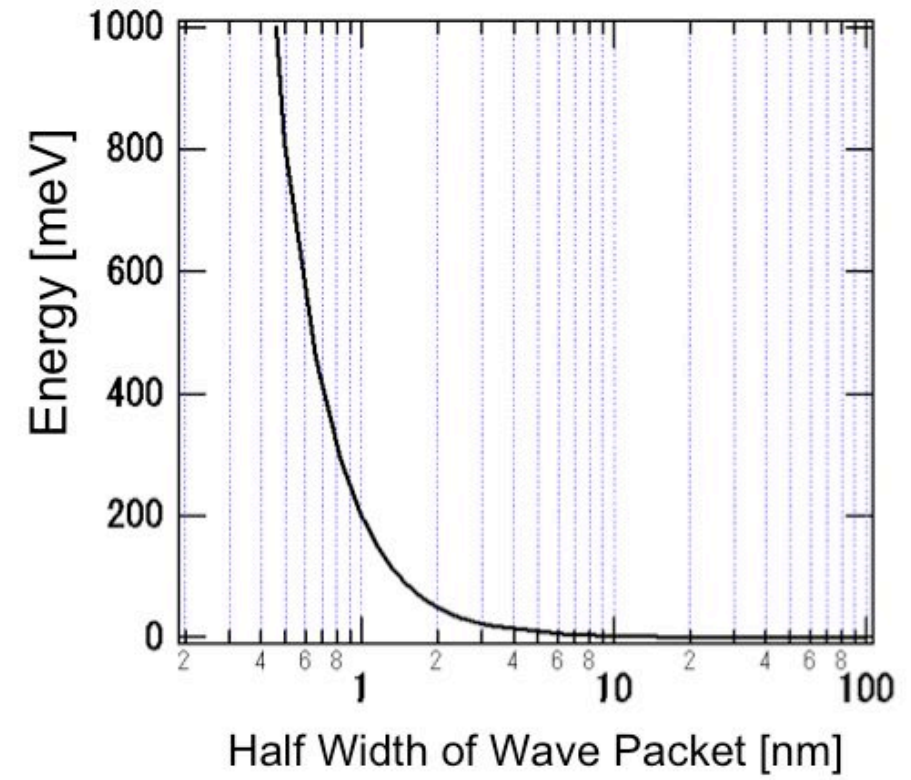

Fig. 4

Fig. 4. Energy of a Gaussian-type wave packet plotted as a function of a half-width of a Gaussian-type wave packet. 